\title{
Hedgehog/Notch-induced premature gliogenesis represents a new disease mechanism for Hirschsprung disease in mice and humans
}

\author{
Elly Sau-Wai Ngan,1,2 Maria-Mercè Garcia-Barceló,1,2 Benjamin Hon-Kei Yip,1,2,3 Hiu-Ching Poon,1 \\ Sin-Ting Lau, ${ }^{1}$ Carmen Ka-Man Kwok, ${ }^{1}$ Eric Sat, ${ }^{1}$ Mai-Har Sham, ${ }^{2,4}$ Kenneth Kak-Yuen Wong,, \\ Brandon J. Wainwright, ${ }^{5}$ Stacey S. Cherny, ${ }^{3}$ Chi-Chung Hui, 6,7 Pak Chung Sham, ${ }^{2,3}$ \\ Vincent Chi-Hang Lui, ${ }^{1,2}$ and Paul Kwong-Hang Tam ${ }^{1,2}$
}

${ }^{1}$ Department of Surgery, ${ }^{2}$ Centre for Reproduction, Development and Growth, ${ }^{3}$ Department of Psychiatry, and ${ }^{4}$ Department of Biochemistry, Li Ka Shing Faculty of Medicine, University of Hong Kong, Pokfulam, Hong Kong, China. Institute for Molecular Bioscience, The University of Queensland, St. Lucia, Australia. ${ }^{6}$ Program in Development and Stem Cell Biology, The Hospital for Sick Children, University of Toronto, Toronto Medical Discovery Towers, Toronto, Ontario, Canada. ${ }^{7}$ Department of Molecular and Medical Genetics, University of Toronto, Toronto Medical Discovery Towers, Toronto, Ontario, Canada.

\begin{abstract}
Hirschsprung (HSCR) disease is a complex genetic disorder attributed to a failure of the enteric neural crest cells (ENCCs) to form ganglia in the hindgut. Hedgehog and Notch are implicated in mediating proliferation and differentiation of ENCCs. Nevertheless, how these signaling molecules may interact to mediate gut colonization by ENCCs and contribute to a primary etiology for HSCR are not known. Here, we report our pathway-based epistasis analysis of data generated by a genome-wide association study on HSCR disease, which indicates that specific genotype constellations of Patched (PTCH1) (which encodes a receptor for Hedgehog) and delta-like $3(D L L 3)$ (which encodes a receptor for Notch) SNPs confer higher risk to HSCR. Importantly, deletion of Ptch1 in mouse ENCCs induced robust Dll1 expression and activation of the Notch pathway, leading to premature gliogenesis and reduction of ENCC progenitors in mutant bowels. Dll1 integrated Hedgehog and Notch pathways to coordinate neuronal and glial cell differentiation during enteric nervous system development. In addition, Hedgehog-mediated gliogenesis was found to be highly conserved, such that Hedgehog was consistently able to promote gliogenesis of human neural crest-related precursors. Collectively, we defined PTCH1 and DLL3 as HSCR susceptibility genes and suggest that Hedgehog/Notch-induced premature gliogenesis may represent a new disease mechanism for HSCR.
\end{abstract}

\section{Introduction}

Hirschsprung (HSCR) disease is the most common neurocristopathy in humans, affecting 1:5,000 newborns. It is a complex genetic disorder attributed to a failure of the enteric neural crest cells (ENCCs) to form ganglia in the hindgut. Colonization of the gut by ENCCs depends on synchronization and balance of the signaling networks involved in the proliferation, migration, and differentiation of ENCCs (1). Hedgehog (Hh) and Notch are known signaling pathways implicated in these processes.

Hh signal transduction is mediated by 2 transmembrane proteins: Smoothened (Smo) is the obligatory signal transducer, and the Hh-binding receptor Patched (Ptch1) constitutively blocks Smo activity in the absence of Hh. Hh binding to Ptch1 unleashes Smo activity and activates the downstream cascade by blocking the formation of the repressor form of the GLI-Kruppel family member GLI1 (Gli1) family of zinc-finger transcription factor, Gli1 ${ }^{\mathrm{R}}$, and promoting the formation of Gli1 activators, $\operatorname{Gli1}^{\mathrm{A}}(2,3)$. It is known that Sonic Hh (Shh) signaling is essential for diverse developmental processes and implicated in various diseases, particularly, those affecting the nervous system and cancer (4-8). Both graded activity and stepwise regulation of Hh signaling are important for its actions. Importantly, mice lacking either the Indian $\mathrm{Hh}-$ or Shh-

Conflict of interest: The authors have declared that no conflict of interest exists. Citation for this article: J Clin Invest. 2011;121(9):3467-3478. doi:10.1172/JCI43737. secreted proteins show partial intestinal aganglionosis, accompanied by megacolon or ectopic ganglia formation, respectively (9), suggesting the biological relevance of these molecules in enteric nervous system (ENS) development.

Similarly, Notch signaling also plays a key role in various aspects of neurogenesis of ENCCs. Activation of Notch signaling is required not only for the maintenance of ENCCs (10) but also for the switching of these cells from a program of neurogenesis to gliogenesis in vitro (11) and in vivo (12). Notch receptors, Notch1 and Notch2, and their ligands, delta-like 1 and delta-like 3 (Dll1 and Dll3), are the main forms expressed in the ENCCs (ref. 10 and our unpublished observations). Notch ligands activate Notch signaling in neighboring cells by inducing a series of proteolytic cleavages of the receptor. The resulting Notch intracellular domain (NICD) translocates to the nucleus in which it associates with the DNAbinding protein CSL (RBP-Jк) to initiate the transcription of target genes, such as hairy and enhancer of split-1 (Hes1) (13), which in turn, represses the expression of proneural proteins (e.g., Mash1) to switch differentiation from neurogenesis to gliogenesis.

Genetic alterations in these signaling molecules may therefore interfere with the colonization process and potentially represent a primary etiology for HSCR. Theoretically, HSCR could be caused by deleterious mutations in a critical signaling component and/or cumulative effects of multiple less severe mutations in these pathways. Nevertheless, so far, none of the genes involved in Hh and 


\section{Table 1}

Genetic interaction between PTCH1 and DLL3 SNPs and summary of subject statistics

\begin{tabular}{|c|c|}
\hline \multicolumn{2}{|c|}{ PTCH1 (Chr9q22.32) } \\
\hline rs357552 ( $\underline{A} / G)$ & rs10512248 (A/C) \\
\hline$\underline{A G}$ or $\underline{A A}$ & AA \\
\hline$\underline{A} G$ or $\underline{\overline{A A}}$ & $A \underline{C}+\underline{C C}$ \\
\hline $\mathrm{GG}$ & $A A$ \\
\hline$\underline{A G}$ or $\underline{A A}$ & $A A$ \\
\hline
\end{tabular}

DLL3 (Chr19q13.2)
rs2354225 (ㅁ/G)
$G G$
$G \underline{A}+\underline{A A}$
$G \underline{A}+\underline{A A}$
$G \underline{A}+\underline{A A}$

MDR identified that 2 SNPs in PTCH1, rs357552 and rs10512248, and 1 SNP in DLL3, rs2354225, were the most relevant on driving the correlation of Hh and Notch pathways. Shown are specific genotype constellations of PTCH1 and DLL3 SNPs, with risk alleles (for rs357552, A; for rs10512248, C; for rs2354225, A) denoted underlined. Case/control frequencies and corresponding risks for HSCR are also shown; genotype constellations are listed in descending order according to risk. Other low-risk genotypes returned a case/control frequency of 31.80/53.90.

\section{Case/control frequency (\%)}

$24.85 / 15.40$

$24.28 / 15.60$

$9.25 / 6.19$
OR (95\% Cl)

$2.78(1.717-4.514)$

$2.64(1.627-4.281)$

$2.53(1.277-5.021)$

$1.82(0.958-3.440)$
Notch pathways were found to be associated with HSCR, neither in a genome-wide association study (GWAS), nor in a mutation screening conducted on patients with HSCR (14). In this study, we further exploited the data initially generated by our GWAS by applying canonical correlation analysis (CCA) to assess whether interactions among gene members of the Hh and Notch signaling pathways could contribute to HSCR. In addition, the coordination mechanism between the Hh and Notch pathways in ENS development has also been examined using Ptch1 $1^{-/-}$mice. To our knowledge, this is the first time that the integrated effect of HhNotch has been demonstrated in HSCR development. Our data may explain the missing heritability of the disease and help identify a new disease mechanism for HSCR.

\section{Results}

Specific genotype constellations of PTCH1 and DLL3 SNPs confer higher risk to HSCR. To assess whether gene-gene interactions in the $\mathrm{Hh}$ and Notch pathways contribute to HSCR, CCA was used to assess epistasis on data sets generated from a GWAS. CCA estimated the association among sets of markers in gene-pairs formed by gene members of the Hh (7 genes) and the Notch (10 genes) signaling pathways (Supplemental Table 1; supplemental material available online with this article; doi:10.1172/JCI43737DS1). Our results indicated that PTCH1 (a Hh receptor) and DLL3 (a Notch ligand) had the largest gene-gene correlation difference (canonical correlation $[\mathrm{Rc}] ; \mathrm{Rc}[\mathrm{cases}]=0.556 ; \mathrm{Rc}$ [controls] $=0.235$; Rc [ difference $]=$ $0.556-0.235=0.321 ; P=0.009)(1,000$ permutations $)$. After adjustment for population stratification (EIGENSTRAT), the $P$ value was still highly significant $(P=0.008)$. Correlations among genes for each group are illustrated in Supplemental Table 2. Given the relevance of ret proto-oncogene (RET) SNPs in HSCR (14), we investigated whether the epistasis identified was dependent on $R E T$ using partial CCA. The $P$ value was 0.014 , indicating that the epistasis was RET independent.

To identify the DLL3 and PTCH1 SNPs driving the correlation, we then used multifactor-dimensionality reduction (MDR). This revealed that 2 out of the 12 PTCH1 SNPs (rs357552 [ $\mathrm{A} / \mathrm{G}]$ and rs10512248 [A/C] ; throughout, underlining indicates a risk allele) and 1 out of the 4 SNPs in DLL3 (rs2354225 [ㅁ/G]) were the most relevant. These SNPs map to putative gene-regulatory regions. Specific genotype constellations of these 3 SNPs conferred higher risk to disease (odds ratio $[\mathrm{OR}]=2.582$ under an additive model and $\mathrm{OR}=2.220$ under a dominant model $[P=0.0003])$ (Table 1 ). Indeed, our patient population was enriched with individuals with risk constellations $(68.2 \%)$ when compared with controls $(46.1 \%)$.
Notch-dependent Hh activity in ENCCs. In light of our genetic and expression data, we proceed to investigate the potential functional interaction of these 2 pathways in ENS development. We and others have previously demonstrated that ENCCs express Hh and Notch signaling components, including Ptch1, Dll1, and Dll3, and that $\mathrm{Hh}$ and Notch signaling are required for the maintenance of the ENCCs by promoting growth and attenuating premature neuronal differentiation $(10,15)$.

In this study, we further examined the potential functional cross-talk between $\mathrm{Hh}$ and Notch signaling in ENS development. Like in other systems, Shh or its agonist (purmorphamine) activated the Ptch1-Smo-Gli1 pathway to promote the proliferation of ENCCs $\left(\mathrm{Ret}^{+} /\right.$neuron-specific class III $\beta$-tubulin $/$ glial fibrillary acidic protein ${ }^{-}\left[\operatorname{Ret}^{+} /\right.$Tuj1-/GFAP- $^{-}$) (Supplemental Figure 1). To assess whether induction of proliferation by Shh is caused by activation of Notch signaling, ENCCs isolated from wild-type mice were treated with Shh in presence or absence of an inhibitor of $\gamma$-secretase, DAPT, which suppresses proteolysis-dependent activation of endogenous Notch receptors (16). Consistent with the above finding, Shh could significantly promote the proliferation of ENCCs, while DAPT treatment counteracted the Shh-induced proliferation (Figure 1, A and B). Thus, Hh-induced proliferation is Notch dependent. In concordance with this data, RT-PCR analysis demonstrated that Shh promotes the expression of a Notch ligand, Dll1, and upregulates the expression of a principle effector of Notch pathway, Hes1 (Figure 1, C-E). Cotreatment of ENCCs with DAPT and Shh caused a similar induction of Dll1 expression as that seen with Shh alone; by contrast, Shh-mediated induction of Hes 1 was suppressed (Figure 1C). This observation suggests that Shh directly upregulates Dll1, while induction of Hes 1 expression is the concomitant of Notch activation. Consistently, as illustrated in the quantitative RT-PCR analyses, Shh-induced expression of Dll1 and Hes 1 could be mimicked by the Shh agonist, purmorphamine (Figure 1, D and E). It is noteworthy that upregulation of Dll3 was not observed in mouse ENCCs (data not shown). Besides the chemical inhibition, we also suppressed the Dll1 expression in ENCCs by siRNA knockdown. Knockdown of Dll1, but not Hes1, with siRNA in ENCCs significantly abolished Hh-induced proliferation, further indicating that $\mathrm{Hh}$ activates the Notch pathway by upregulating Dll1 expression in ENCCs (Figure 1F and Supplemental Figure 2).

Deletion of Ptch1 activates Hh signaling and promotes proliferation of ENCCs. Concordant results were independently obtained from Ptch1 $1^{-/-}$ENCCs. At E11.5, ENCCs were isolated from embryonic guts of floxed Ptch1 mice (Ptch1f/f mice), which have Cre recombinase 
A

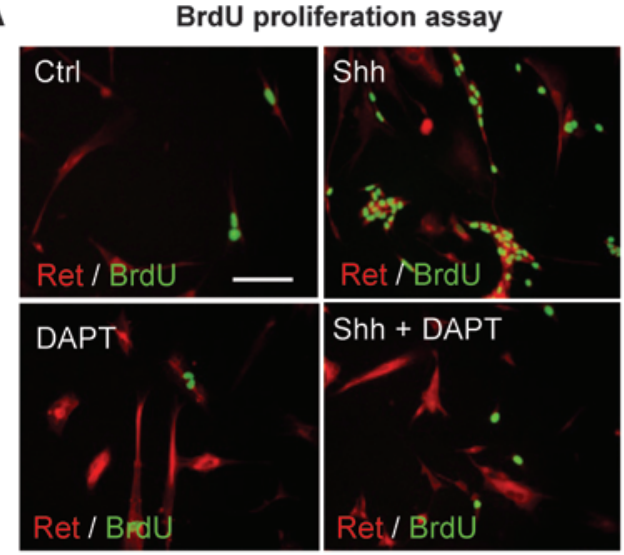

B

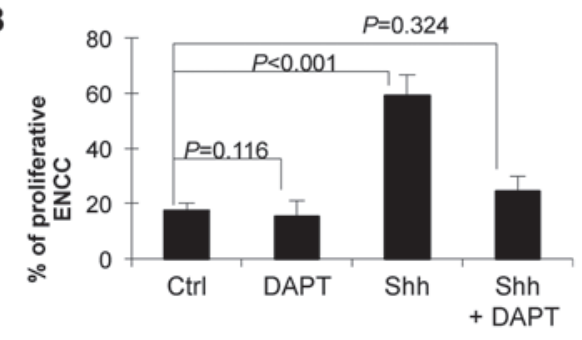

C

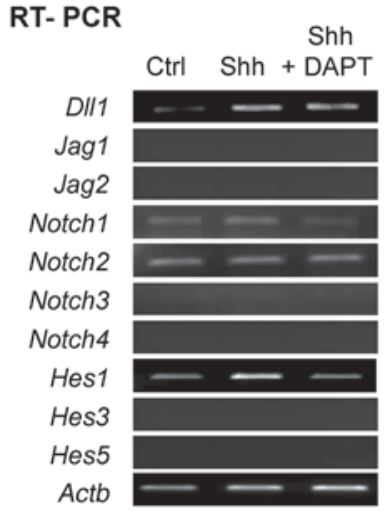

D

qRT-PCR

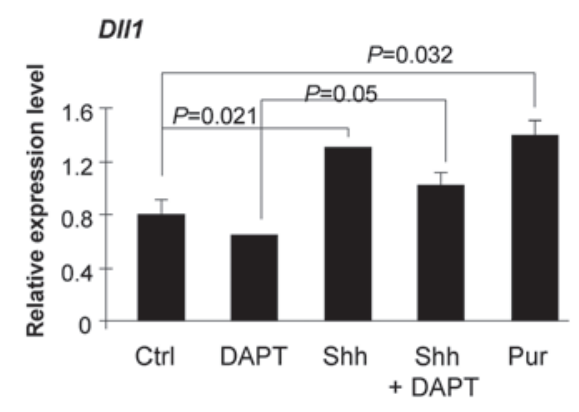

E

qRT-PCR

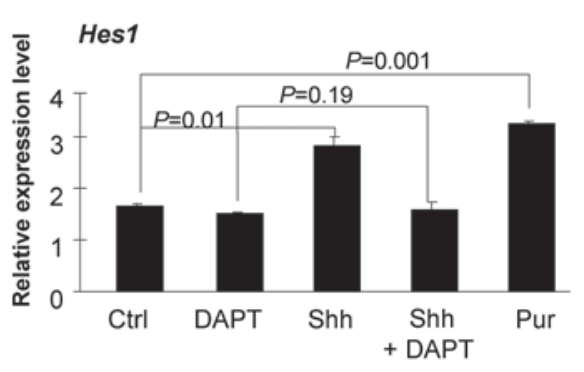

$\mathbf{F}$

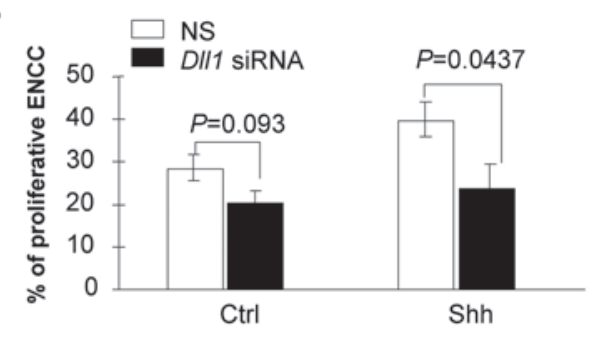

qRT-PCR

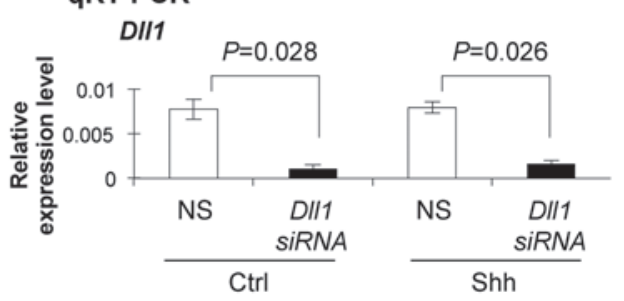

Figure 1

Shh upregulates DII1 expression to mediate proliferation of ENCCs. (A) BrdU proliferation assay showed that Shh (3 days) significantly $(P<0.001)$ increases the proliferation of ENCC progenitors (BrdU+/Ret+). This effect could be counteracted by treatments with DAPT (10 mM). Scale bar: $100 \mu \mathrm{m}$. Ctrl, control. (B) Proliferating Ret-expressing cells that incorporated with BrdU (for 16 hours) were counted. The values reported in bar charts represent the mean \pm SEM, and 3 independent experiments were performed. Data were analyzed by 1-way ANOVA, followed by the Tukey post-hoc test. $P$ values of less than 0.05 are statistically different from those of the control. (C) Semiquantitative RT-PCR for the Notch pathway genes after treatment with Shh in presence or absence of the Notch inhibitor DAPT (10 mM) (3 days). (D and E) Quantitative RT-PCR (qRT-PCR) for the expression of DII1 and Hes1 in ENCCs treated with Shh, Shh plus DAPT, and Hh agonist, purmorphamine (Pur, $1 \mathrm{mM}$ ) (3 days). (F) Knockdown of DIl1 significantly abrogated the Shh-mediated proliferation (3 days). Quantitative RT-PCR for the expression of DII1 shows that siRNA can significantly downregulate DII1 expression (3 days). Nonsilencing RNA (NS) was used as a negative control. Bars represent the mean \pm SEM, and 3 independent experiments were performed, each in triplicate. $P$ values of less than 0.05 were considered to be statistically significant.

recognition $($ loxP $)$ sites flanking exon 3 of the Ptch1 gene (ref. 17 and Figure 2A). Ptch1f/f ENCCs were then infected with Cre-encoding adenovirus (Ad-GFP-Cre) to delete exon 3 of the Ptch1 gene; the expression of the mutant Ptch1 and other Hh target genes was analyzed by RT-PCR. The majority of ENCCs were expressing GFP 72 hours after the infection with the control virus (Ad-GFP) and AdGFP-Cre (Figure 2A). Consistently, RT-PCR also indicated that Cre adenovirus can completely delete exon 3 of the Ptch1 gene in ENCCs to generate the null mutant form of Ptch1 (Figure 2B). Given that Ptch1 is a negative regulator of Smo, inactivation of Ptch1 constitu- 
A Ptch1 $1^{\text {tff }}$

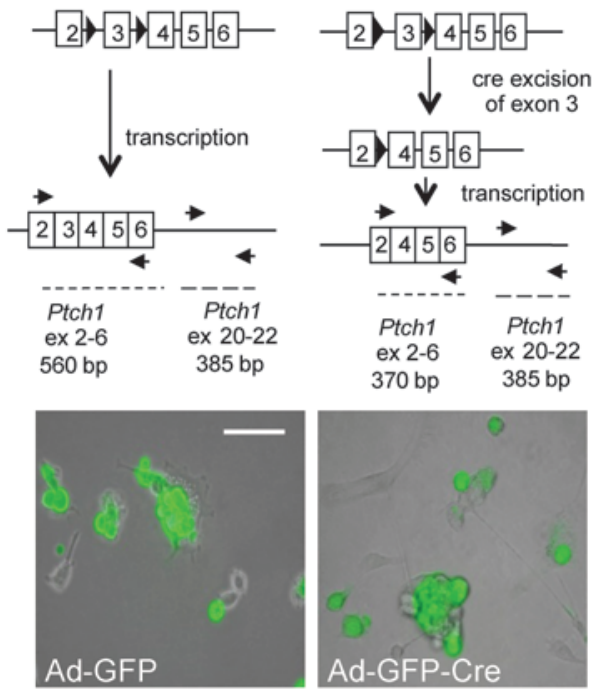

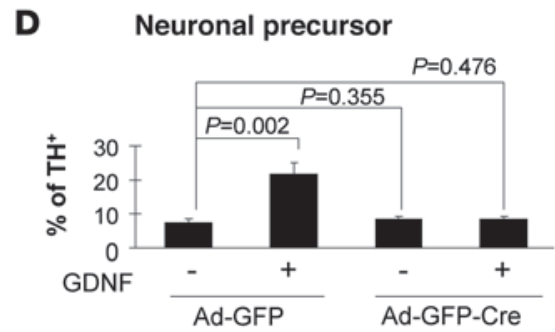

E

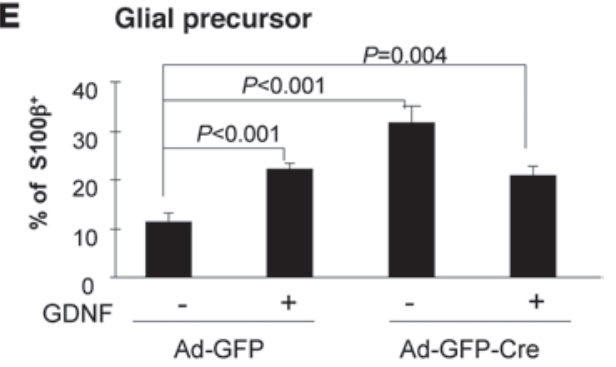

F $\quad$ qRT-PCR

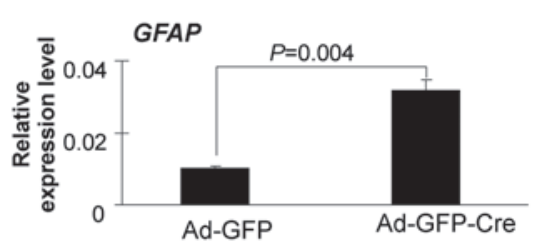

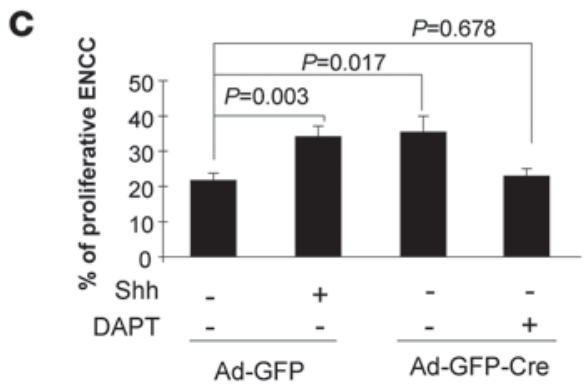

Figure 2

Deletion of Ptch1 promotes the proliferation and gliogenesis but inhibits neurogenesis of ENCCs. (A) ENCCs were isolated from the Ptch $1^{f / f}$ mice, which have loxP recombinase recognition sites flanking exon 3 of the Ptch1 gene. The Ptch $1^{f / f}$ ENCCs were then infected with Ad-GFPCre. $86.0 \% \pm 1.3 \%$ of ENCCs expressed GFP 72 hours after the infection with the control virus (Ad-GFP) and Ad-GFP-Cre. Primers were designed to generate a PCR product that spanned exon 2 to 6 (ex 2-6) of Ptch1 to distinguish full-length and exon 3-deleted Ptch1 transcripts. Another pair of primers was designed at exon 20 and 22 for the comparison of Ptch1 expression. Exons are represented by rectangles, and loxP sites are represented by triangles. Scale bar: $100 \mu \mathrm{m}$. (B) The expression of the mutant Ptch1 and other Hh target genes was analyzed by RT-PCR in presence or absence of Shh (transduction for 4 days followed by Shh treatment for 3 days). (C) BrdU proliferation assay. Relative proliferation rates were measured by counting BrdU $+/ \mathrm{Ret}^{+}$and total Ret $^{+}$cells. (D and E) Immunocytochemistry. Neuronal and glial differentiation was monitored based on the expression of TH and S100 $\beta$, respectively (GDNF treatment for 10 days). Percentages of (D) neuronal and (E) glial precursors were measured over the total number of ENCCs (DAPI). (F) Quantitative RT-PCR showed that deletion of Ptch1 (Ad-GFP-Cre) induces GFAP expression. The values reported in bar charts represent the mean \pm SEM, and 3 independent assays were performed. Data were analyzed by 1 -way ANOVA, followed by the Tukey post-hoc test. $P$ values of less than 0.05 were considered to be statistically significant.

tively activates the Hh pathway in ENCCs similar to that observed in control ENCCs treated with Shh. Cre adenovirus-mediated deletion of Ptch1 (Ad-GFP-Cre) resulted in an elevated expression of Gli1, accompanied by an extensive proliferation of ENCCs. It was similar to the control virus-infected ENCCs with Shh treatment (Figure 2, B and C). Addition of Shh to Ptch1-/- ENCCs did not fur- ther increase the expression of Gli1 (Figure 2B) or their proliferation (Supplemental Figure 3), suggesting that Ptch1 deletion is sufficient to fully activate $\mathrm{Hh}$ signaling. A similar counteracting effect of DAPT was also observed in Ptch $1^{-/}$ENCCs, indicating that activation of Notch by Shh or by deletion of Ptch1 in ENCCs is required for the observed elevated proliferation (Figure 2C). 
A

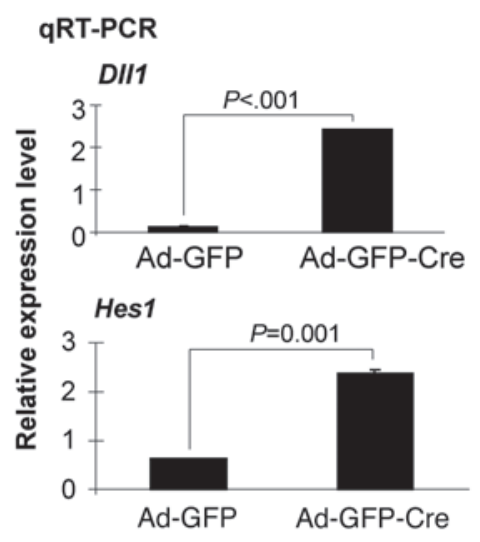

B

WB
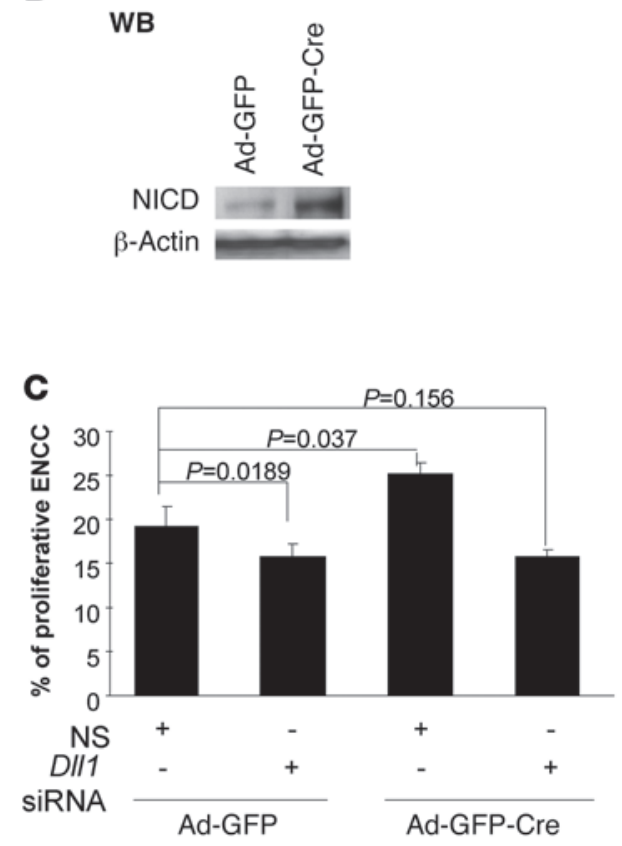

D
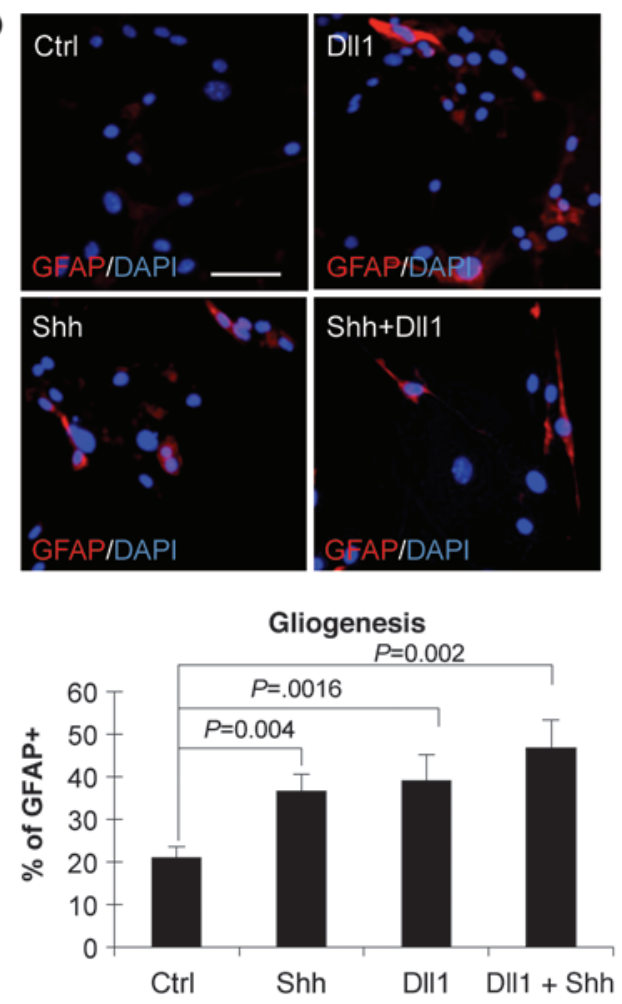

$\mathbf{E}$

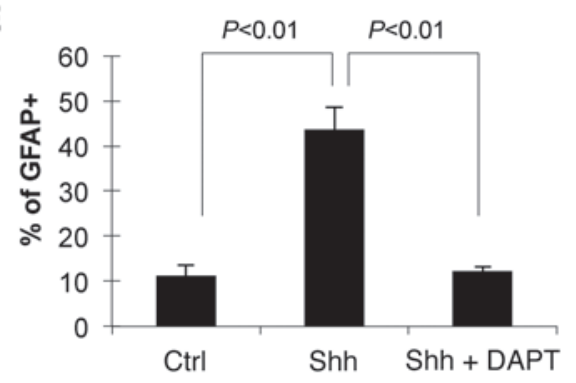

\section{Figure 3}

Deletion of Ptch1 in ENCCs robustly activates the Notch pathway. (A) Quantitative RT-PCR showed that Cre adenovirus-mediated deletion of Ptch1 in ENCCs (Ad-GFP-Cre) resulted in elevated expression of DII1 and Hes1 compared with that of the cells infected with control virus (Ad-GFP) (4 days). (B) Western blot analysis showed that deletion of Ptch1 induces a robust expression of NICD. (C) BrdU proliferation assay indicated that deletion of Ptch1 (Ad-GFP-Cre) promotes the proliferation of ENCCs, while knockdown of DII1 using siRNA (3 days) significantly reduces the proliferation of Ptch1--- ENCCs. Nonsilencing RNA was used as a negative control. Error bars indicated mean \pm SEM, and 3 independent experiments were performed. (D) Immunocytochemistry showed that Shh or DII1 alone is sufficient to induce the gliogenesis of ENCCs. ENCCs were treated with Shh $(2 \mu \mathrm{g} / \mathrm{ml})$, Dll1 $(1 \mu \mathrm{g} / \mathrm{ml})$, or a combination for 8 days, and cells were fixed and subjected to immunostaining with anti-GFAP antibody. GFAP+ (red) cells are glia. Scale bar: $100 \mu \mathrm{m}$. (E) Immunocytochemistry analyses indicated that addition of DAPT significantly abolishes Hh-mediated glial differentiation. ENCCs were treated with Shh (2 $\mu \mathrm{g} / \mathrm{ml})$, DAPT $(10 \mathrm{mM})$, or a combination for 8 days, and cells were fixed and subjected to immunostaining with anti-GFAP antibody. Quantitative data are shown in bar chart. Error bars indicated mean \pm SEM, and 3 independent experiments were performed. Data were analyzed by 1 -way ANOVA followed by the Tukey post-hoc test. $P$ values of less than 0.05 were considered to be statistically significant.

Deletion of Ptch1 in ENCCs inhibits neurogenesis and is accompanied by spontaneous gliogenesis. To examine whether deletion of Ptch1 affects the differentiation capacity of ENCCs, we induced the neuronal differentiation of ENCCs with the administration of glial cell-derived neurotrophic factor (GDNF). Neuronal and glial differentiation were monitored based on the expression of tyrosine hydroxylase
$(\mathrm{TH})$ or Tuj1 and S100 $\beta$, respectively. The control virus-infected ENCCs gave rise to both neurons and glia in the presence of GDNF (Figure 2, D and E). Conversely, deletion of Ptch1 in ENCCs resulted in a significant reduction of $\mathrm{TH}^{+}\left(\right.$Figure 2D) and Tuj $1^{+}$(Supplemental Figure 4) cells upon the GNDF treatment. In addition, spontaneous gliogenesis was observed in $\mathrm{Ptch}^{-/-}$ENCCs, and a 
A
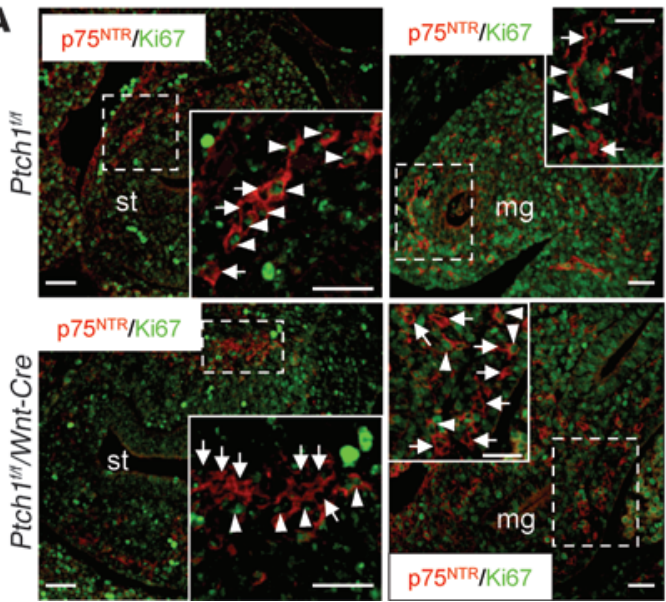

B

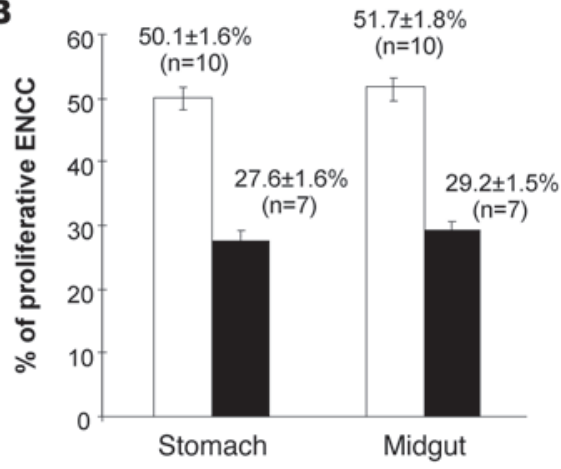

Ptch $1^{\text {ff }}$

Ptch1 $1^{f f}$ Wnt1-Cre

\section{Figure 4}

Sustaining Hh signaling reduces proliferative ENCCs in mice. (A) Proliferation of ENS progenitors in E11.5 control $\left(P\right.$ tch $\left.1^{f / f}\right)$ and conditional Ptch1 knockout (Ptch1ff/Wnt1-Cre) guts was analyzed by immunofluorescence for Ki67 (green) and p75NTR (red). Proliferating ENCCs (p75 ${ }^{\mathrm{NTR}+}$, Ki67+; arrowheads) and nonproliferating ENCCs (p75 ${ }^{\mathrm{NTR}+}$, Ki67-; arrows) were identified and counted in control and mutant guts. Boxed regions are shown at higher magnification in insets. st, stomach; mg, midgut. (B) The percentages of proliferative ENCCs in control and mutant guts were calculated and are shown in the bar chart. Error bars indicated \pm SEM across 10 control $\left(P t c h 1^{f / f}\right)$ and 7 mutant (Ptch1/f/Wnt1-Cre) mice. Scale bar: $50 \mu \mathrm{m}$.

significantly greater percentage of cells were $S 100 \beta^{+}$in the absence of GDNF (Figure 2E). GDNF treatment of these glia-committed ENCCs, on the other hand, slightly reduced the percentage of $\mathrm{S} 100 \beta^{+}$cells in the culture, suggesting that it may exhibit a negative regulatory effect on their spontaneous gliogenesis (Figure 2E). Nevertheless, GDNF treatment alone was not sufficient to fully retrieve the neuronal differentiation capacity of $P t c h 1^{-/-}$ENCCs (Figure 2E). Consistently, the expression of GFAP was significantly upregulated, as illustrated by quantitative RT-PCR (Figure 2F).

Dll1 integrates Hh and Notch pathways in ENCCs. Constitutive activation of Hh signaling in $\mathrm{Ptch}^{-/-}$ENCCs also induced robust expression of Dll1, Hes1 (Figure 3A), and the NICD (Figure 3B). Again, Dll1 served to integrate $\mathrm{Hh}$ and Notch pathways to promote the Hh-mediated proliferation and glial differentiation. Knockdown of Dll1 in Ptch1 $1^{-/}$ENCCs abolished the Hh-induced proliferation (Figure 3C), while Dll1 mimicked Shh and was sufficient to drive ENCCs to a glial lineage (Figure 3D). Consistently, Hh-induced gliogenesis could be abolished by addition of Notch inhibitor (DAPT) (Figure 3E). Together, these data suggest that constitutive activation of Hh signaling robustly activated the Notch pathway via upregulation of Dll1 to promote glial differentiation.

Aberrant Hh signaling reduces enteric progenitors and induces premature gliogenesis in mouse embryonic guts. To study the effect of aberrant Hh signaling in premigratory ENCCs, we generated neural crest-specific Ptch 1 knockout mice by crossing Ptch $1^{\text {f/f }}$ mice with Wnt1-Cre transgenic mice, which express Cre recombinase specifically in the neural crest, including premigratory ENCCs (10). Neural crest-specific Ptch1 mutant mice died at E12 and exhibited a significant $(P<0.001)$ reduction of proliferative ENCCs $\left(\mathrm{p} 75^{\mathrm{NTR}+} / \mathrm{Ki} 67^{+}\right)$in the bowel (Figure 4$)$. In concordance with the in vitro data, significantly more Notch-activated cells $\left(\mathrm{Hes} 1^{+}\right)$ were found in the mutant bowels (Figure 5, A and B), and the expressions of Hes 1 and Dll1 were elevated in the ENCCs isolated from the mutant embryonic guts (Figure 5, C and D). Given that $\mathrm{Ptch1}^{-/-}$ENCCs could migrate to the bowel and cell death was barely detected (Supplemental Figure 5), the reduction of proliferating postmigratory ENCC progenitors would be a consequence of premature gliogenesis of $\mathrm{Ptch}^{1^{-/-}}$ENCC progenitors.

Given that gliogenesis starts at E12-E12.5 or later, Ptch1ff//Wnt1-Cre embryos did not survive long enough to allow us to examine the effect of Ptch1 deletion on gliogenesis. To overcome this problem, mice with Cre knocked into the Hoxb3 b3-IIIa enhancer region (b3-IIIa-Cre mice) were used to specifically knockout Ptch1 in vagal neural crest cells. As described previously, Cre was only expressed transiently during E9.5-E10.5 in 30\% of vagal neural crest cells (18), so Ptch1 was not completely deleted in these mutants. Nevertheless, these embryos exhibited similar phenotypes to those seen in the Ptch1f/f/Wnt1-Cre embryos (Supplemental Figure 6). Importantly, E12.5 mutant embryos (Ptch1f/f, b3-IIIa-Cre) could be collected and used to examine whether deletion of Ptch1 causes premature gliogenesis in vivo. As demonstrated in the whole-mount in situ hybridization, the glial marker, fatty acid-binding protein-7 (Fabp7), was barely detected in the control embryonic guts at E12.5, while markedly increased expression of Fabp7 was observed in the mutants (b3-IIIa-Cre, Ptch1f/f) (Figure 6 and Supplemental Figure 7). Taken together, our data showed that deletion of Ptch1 induces an early onset of gliogenesis of ENCCs in mice.

Hh-induced gliogenesis is conserved among mice and humans. Only Dll1, but not Dll3, serves to integrate the Notch and Hh pathways in mouse ENCCs, while DLL3 was found to genetically interact with PTCH1 and contribute to HSCR in humans. These observations prompted us to further examine whether $\mathrm{Hh}$-induced gliogenesis is conserved between mice and humans. To achieve this, human neural crest-like cells, skin-derived precursors (SKPs), were used. SKPs have been shown to express a panel of neural crest markers and to be able to generate all major neural crest derivatives $(19,20)$, including cells that express many markers of enteric neurons (unpublished observations). As illustrated in Figure 7, SKPs isolated from human foreskin samples grew as neurospheres and expressed the stem cell marker, vimentin (Figure 7A). They exhibited high plasticity to form neurons 

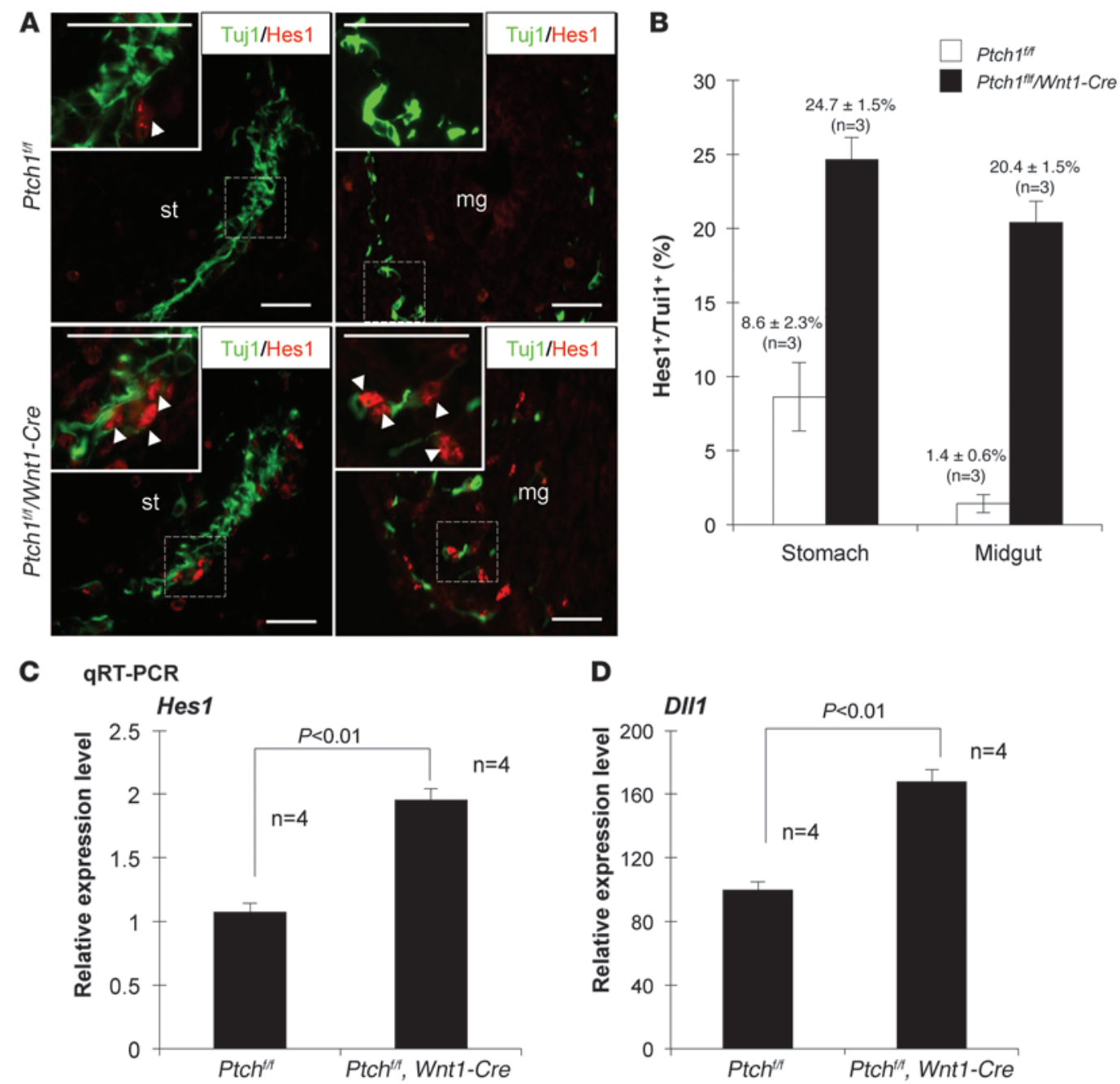

\section{Figure 5}

Deletion of Ptch1 in ENCCs upregulates the expression of Hes1 and DII1. (A) Immunohistochemical analyzes were performed on E11.5

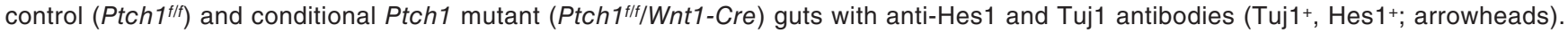
Boxed regions are shown at higher magnification in insets. Scale bar: $50 \mu \mathrm{m}$. (B) The percentages of Hes $1^{+}$cells in controls and mutants were measured over the total number of Tuj $1^{+}$cells and are shown in the bar chart. Error bars indicated \pm SEM across 3 controls $\left(P t c h 1^{t / f}\right)$ and mutants (Ptch1 1f//Wnt1-Cre). (C and D) Quantitative RT-PCR for the expression of (C) Hes1 and (D) DIl1 in ENCCs isolated from E11.5 control $\left(P t c h 1^{f / f}\right)$ and mutants $\left(P t c h 1^{f / f} /\right.$ Wnt1-Cre $)$. Error bars indicated \pm SEM across 4 controls $\left(P t c h 1^{f / f}\right)$ and 4 mutants $\left(P t c h 1^{f / f} /\right.$ Wnt1-Cre $)$.

(Figure 7A, neurofilament) and glia (Figure 7A, GFAP ${ }^{+}$. More importantly, similar to that in mouse ENCCs, Hh could induce expression of the glial protein GFAP in human SKPs (Figure 7B). Treatment with $\mathrm{SHH}$ significantly increased the number of $\mathrm{GFAP}^{+}$cells when compared with those of the untreated controls (Figure 7B).

\section{Discussion}

The ENS in mammals is derived from a small pool of progenitor cells, the ENCCs. These precursor cells extensively proliferate to expand and migrate over a long distance to fully colonize the developing gut and differentiate into millions of neurons and glia, which are organized into the network responsible for coordinating the complex behaviors of the gut (1). This developmental process involves sequential waves of neurogenesis and gliogenesis and requires an appropriate balance between the proliferation and differentiation of ENCCs and their progeny. Therefore, integration of different signaling pathways coordinating proliferation and neuronal/glial differentiation is critical for proper ENS development and defects in any of these pathway components may lead to HSCR.

Genetic screenings on patients with HSCR have identified susceptibility loci and mutations associated with the disease. Mutations in the known HSCR genes, however, can only account for approximately $15 \%-20 \%$ of sporadic cases. Other HSCR cases may therefore likely result from the cumulative effects of less severe ablations in genetic pathways governing ENS development. To identify novel risk loci, our group has recently conducted a GWAS on HSCR. The genotype data generated from GWAS not only allowed us to define the risk loci that we believe to be new but also to detect gene-gene interactions. Here, we have integrated genetics, statistics, and bioinformatics with experimental biology to investigate the mechanisms by which a discrete number of genes interact with each other to cause disease. 
A

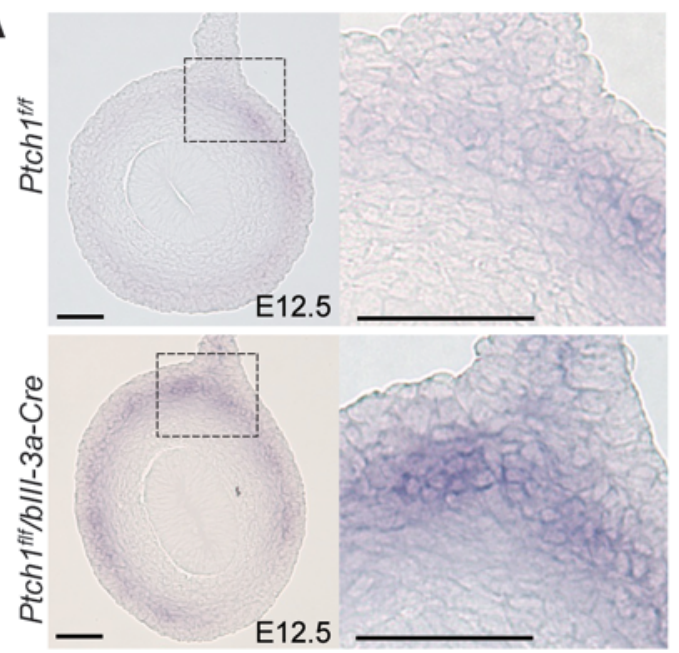

\section{qRT-PCR}

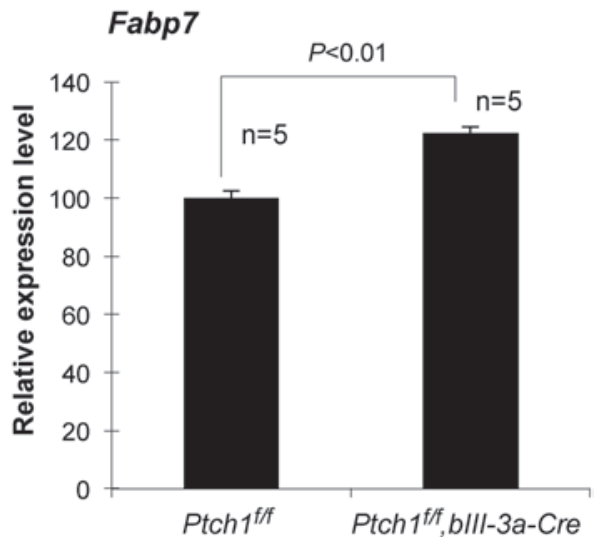

\section{Figure 6}

Ptch1 mutant exhibits premature gliogenesis. (A) In situ hybridization was used to examine the expression of glial marker (Fabp7) on E12.5

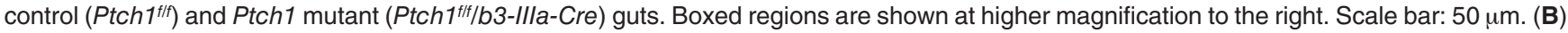
Quantitative RT-PCR data on the expression of Fabp7 in E12.5 control (Ptch1/ff) and mutant (Ptch1/f/Wnt1-Cre) guts is shown in the bar chart. Error bars indicated \pm SEM across 5 control $\left(P t c h 1^{1 / f}\right)$ and 5 mutants $\left(P t c h 1^{f / f} /\right.$ Wnt $\left.1-C r e\right)$. Data was analyzed by Student's $t$ test. $P$ values of less than 0.05 were considered to be statistically significant.

We used CCA to assess whether interactions among gene members of these signaling pathways (Hh and Notch) could contribute to HSCR. To our knowledge, this is the first time that CCA was used to assess epistasis on data sets generated from a GWAS. This approach not only illuminates the genetic architecture of a complex disease but also enhances the potential of genetic variants for risk prediction using GWAS data. To validate our genetic data, we performed functional studies to demonstrate that the gene-gene interaction between $\mathrm{Hh}$ and Notch is not limited at the genetic level. Indeed, there was a functional intercross among these 2 pathways during ENS development. Importantly, both of these 2 independent approaches indicated that the Notch ligand Dll serves to integrate these pathways. Therefore, Dll may play a pivotal role in ENS development and the pathogenesis of HSCR.

It is interesting to note that fewer proliferating ENCC progenitors were observed in the Ptch1 mutant embryonic guts, even though both $\mathrm{Hh}$ and Notch are key signaling pathways implicated in preserving a pool of undifferentiated progenitors $(9,15$, 21). This implies that although proper Hh function is necessary for the formation of sufficient numbers of enteric ganglia in the bowel (9), excess Hh pathway activation in ENCCs, on the other hand, causes progenitor progression (i.e., differentiation). It is known that the ENCC-derived neuronal and glial precursor cells continue to proliferate while progressively differentiating $(22,23)$. Therefore, during the early events of this developmental process, Hh may control the pool sizes of neurons and glia by mediating the proliferation of neural and glial precursors and/or switching neurogenesis to gliogenesis. Given that there was no obvious change in the neuronal precursors in the Ptch1 mutant embryonic guts at E10.5 (data not shown) and E11.5 (Tuj1+, Supplemental Figure 8), the progenitor progression may not be the result of a precise regulation of neuron production (pool size of neuronal precursors). In other words, it is possible that progenitor progression depends upon feedback inhibition and/or feedforward induction mediated by pool size of naive ENCCs. Sus- taining Hh activity may induce a rapid expansion of the ENCC pool, leading to a feedback inhibition of proliferation and subsequently, a feed-forward induction of progenitor progression. Indeed, even though deletion of Ptch1 promotes proliferation, we found that the $P t c h 1^{-1-}$ ENCCs still retain their differentiation capacity to form neurons and glia in vivo (Supplemental Figure 4 and Figure 6). Similar observations have been reported previously in mice with the Ptch1 deletion in granule neuron precursors (GNPs) and neural stem cells (NSCs). While Ptch1-/- GNPs and NSCs exhibited increased proliferation, they still remain capable of differentiating into astrocytes, oligodendrocytes, and neurons (6). Most likely, there is an additional layer of signaling refinement in the gut environment to compensate for the inhibitory effect of Hh in the process of neurogenesis.

Our functional data suggest that activation of Notch would be the feed-forward signaling for progenitor progression. In this respect, activated Notch has been shown to have an iterative role on mitotic and postmitotic precursor cells in specifying or promoting a particular cell fate, notably glia cell fate during nervous system development $(11,24,25)$. Notch activity permits the early progenitor cells to remain proliferative and undifferentiated, and downregulation of Notch activity is required for the cells to exit the cell cycle. Subsequent reactivation of the Notch pathway mainly regulates the neuronal versus glial fate choice (26). This temporary release from Notch activation allows resumption of the production of temporally appropriate neurons and is crucial for proper glial differentiation $(26,27)$. Therefore, constitutive activation of the Hh pathway in ENCCs causes sustained Notch activity. Absence of a release from Notch activation may result in incomplete neuronal differentiation and premature gliogenesis (Supplemental Figure 9).

Our initial GWAS has already defined the glial mediator neuregulin 1 (NRG1) as a major risk locus for HSCR aside from SNPs in RET (14). Our current study further highlighted the relevance of genes governing gliogenesis in the pathogenesis of HSCR. In 
A
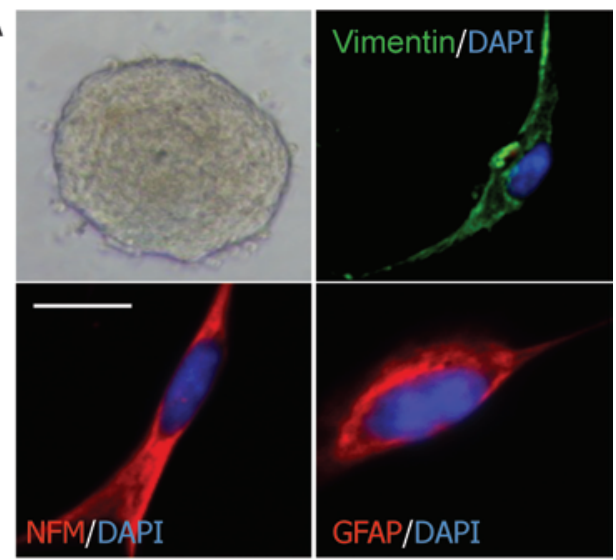

B

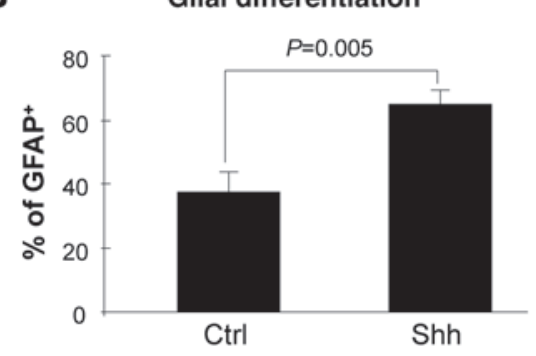

summary, we have identified HSCR susceptibility genes and specific genotype constellations of PTCH1 (a Hh receptor) and DLL3 (a Notch ligand) SNPs that conferred higher risk to HSCR. In addition, we provided definitive in vivo evidence of the essential role played by the interaction between $\mathrm{Hh}$ and Notch pathways in coordinating neuronal and glial cell differentiation during ENS development. We also defined a molecular mechanism in which Dll integrates Hh and Notch pathways to promote glial differentiation. In concordance with our initial GWAS, our current data further indicated that premature gliogenesis may represent a new disease mechanism for HSCR, and Hh-Notch-induced premature gliogenesis may lead to HSCR.

\section{Methods}

\section{Epistasis analysis}

A GWAS on HSCR was previously conducted with 173 cases and 436 controls using Affymetrix $500 \mathrm{~K}$ chips (14). Gene-gene interaction was interrogated on genes members of the Hh and Notch signaling pathways (Supplemental Table 1).

We decided to implement CCA, because it is the most general of multivariate techniques (28). It is also a natural statistical framework to study 2 sets of variables and see what is common among the 2 sets. CCA differs from existing methods for gene-gene interaction detection (i.e., logistic regression) in that it simultaneously considers all markers in the genes to be correlated. This can both reduce the multiple-testing burden and increase statistical power. CCA estimates the maximum correlation of 2 canonical variables, where each canonical variable is a set of linear combinations of SNPs from 1 gene. In general, there may exist more than one significant canonical correlation; however, the first canonical correlation is always the one explaining most of the relationship between the genes tested. In this study, the CCA among genes

\section{Figure 7}

Hh induces gliogenesis of SKPs. (A) SKPs isolated from human foreskin could give rise to cells expressing neuronal and glial markers. SKP spheres were obtained 2 to 3 weeks after culture. Immunocytochemistry showed that SKPs express precursor marker (vimentin, green). Two weeks after culturing in the differentiation medium, cells expressing neuronal (neurofilament [NFM+], red) and glial (GFAP, red) markers were observed. Cells were counterstained with DAPI (blue). Scale bar: $50 \mu \mathrm{m}$. (B) The percentages of glial (GFAP+) cells in untreated control and Shh-treated culture were counted over total number of cells (DAPI). Error bars indicated \pm SEM of triplicates. Data was analyzed by $t$ test. $P$ values of less than 0.05 were considered to be statistically significant.

was obtained for cases and controls separately. Then we calculated the $P$ value (evaluated via permutation) of the differences in gene-pair first canonical correlations between cases and controls. The largest significant correlation difference between patients and controls identified the 2 interacting genes. Correlations between genes for each group are illustrated in Supplemental Table 2.

To correct for population stratification, we partitioned out the population effect (EIGENSTRAT) using partial CCA (29). The partial CCA is the natural generalization of partial correlation from the univariate to the multivariate situation. Given 3 vectors of random variables, $X, Y, Z$, CCA allows study of the relationship between $X$ and $Y$ after partitioning out the effect of vector $Z$ on both $X$ and $Y$ vectors (e.g., make the effect of $Z$ constant). While regular CCA uses only the variance-covariance matrix of $X$ and $Y$, the partial CCA uses the conditional variance-covariance matrix of $X$ and $Y$ given $Z$ (e.g., the residualized variance-covariance matrix) to partition out the effect of $Z$. Partial CCA was also used to investigate whether the epistasis identified between $\mathrm{Hh}$ and Notch was dependent on RET SNPs (rs2082106, rs2742234, rs17028). We next stratified the patients and controls according to the high-risk or low-risk genotypes generated by MDR to assess the relative risk to HSCR in terms of OR (30). The EIGENSOFT package and the MDR program were used.

\section{Mice}

Ptch1fff, Wnt1-Cre, and b3-IIIa-Cre mice have been described previously (17, 18, 31). Swiss mice (Canadian CD-1 [ICR]) were from Charles River Laboratories. Animals were maintained in the Laboratory Animal Unit at the University of Hong Kong, and all experiments were performed in accordance with procedures approved by the committee on the Use of Live Animals, the University of Hong Kong (CULTRA 1998-09).

\section{ENCC culture}

All ENCCs were isolated from E11.5 ICR, Ptch 1f/f, or Ptch1f/f/Wnt1-Cre mouse embryonic guts (from stomach to hindgut), and guts were dissected in L15 medium (Invitrogen). Guts were washed with $\mathrm{Ca}^{2+}$ - and $\mathrm{Mg}^{2+}$ free PBS and digested with collagenase/dispase $\left(0.2 \mu \mathrm{g} / \mathrm{ml}\right.$ each; $37^{\circ} \mathrm{C}$ for 10 minutes). Digested guts were triturated into single cells and filtered through cell strainers $(100-\mu \mathrm{m}$ strainers and then $40-\mu \mathrm{m}$ strainers, Falcon, $\mathrm{BD}$ Biosciences). Cells were resuspended in neural crest medium, DMEM containing $15 \%$ chick embryo extract (SIL), FGF (20 ng/ml, SigmaAldrich), EGF (20 ng/ml, Sigma-Aldrich), Retinoic acid (35 ng/ml, SigmaAldrich), N2 (1\%), B27 (2\%, Invitrogen), and $\beta$-mercaptoethanol (50 mM, Sigma-Aldrich); plated onto poly-D-lysine-coated and fibronectin-coated $(20 \mu \mathrm{g} / \mathrm{ml}$ each, Invitrogen) wells; and enriched by multiple replatings as described previously $(15,32,33)$. The ENCCs at passage 3 were harvested for quantitative RT-pCR or seeded at clonal density $\left(1 \times 10^{5}\right.$ to $1.5 \times 10^{5}$ cells per each $35-\mathrm{mm}$ well and $1.5 \times 10^{4}$ cells per each $20-\mathrm{mm}$ well) and used for the subsequent functional analyses. 


\section{Human SKP culture}

SKPs were prepared from human foreskin as described previously (19). Briefly, dissected skin was minced into 4 - to $5-\mathrm{mm}^{2}$ pieces and then digested with Accumax (Chemicon) for 2 hours at room temperature, followed by overnight incubation with Accumax at $4^{\circ} \mathrm{C}$. After removal of the epidermis, the tissues were cut into 1 - to $2-\mathrm{mm}^{2}$ pieces and further digested with Accumax with collagenase XI ( $1 \mathrm{mg} / \mathrm{ml}$, Sigma-Aldrich) and DNase I $(0.2 \mathrm{mg} / \mathrm{ml}$, Sigma-Aldrich $)$ at $37^{\circ} \mathrm{C}$ for 30 minutes, mechanically dissociated, and filtered through a $70-\mu \mathrm{m}$ cell strainer (Falcon). Dissociated cells were pelleted and plated in DMEM/F12 (3:1; Invitrogen) containing $20 \mathrm{ng} / \mathrm{ml} \mathrm{EGF}$ and $40 \mathrm{ng} / \mathrm{ml} \mathrm{FGF2}$ (both from Sigma-Aldrich). For differentiation assay, SKPs were plated on chamber slides (Nalge Nunc) coated with poly-D-lysine-laminin in DMEM/F12 (3:1) supplement with 10\% FBS. Recombinant human Shh ( $2 \mathrm{mg} / \mathrm{ml}$; C24II, R\&D Systems) was added 1 week after culture and incubated for 7 additional days. Cells were then fixed and stained with specific antibodies as mentioned below.

\section{Viral transduction}

Cre-GFP (Ad-GFP-Cre) or GFP (Ad-GFP) recombinant adenovirus (Vector Biolaboratories) was added to cells in a 1:50 dilution $\left(\sim 1 \times 10^{8} \mathrm{PFU} / \mathrm{ml}\right)$. The infection efficiency was estimated by counting the GFP-positive cells under the fluorescence microscope (Olympus CKX41 Inverted Microscope; Olympus). Seventy-six hours after the infection, approximately $90 \%$ of the cells expressed GFP, and then cells were harvested. Deletion of Ptch1 and activation of the Hh signaling were confirmed by RT-PCR and Western blot as described below. Cell proliferation assays were performed 7 days after infection.

\section{RNA interference}

Dll1 siRNA (catalog no. S100980007, CGTCTATCTTGGATTACTA) and a nonspecific siRNA control (catalog no. 1022079, AATTCTCCGAACGTGTCACGT) (Qiagen) were used. $1.5 \times 10^{5}$ ENCCs were transfected with Dll1 or nonspecific siRNA using LipofectAMINE 2000 (Invitrogen) according to the manufacturer's instructions. After 24 hours, the cells were subjected to cell proliferation assay and RNA extraction in the presence or absence of Shh.

\section{Cell proliferation assay}

Cell proliferation was measured using the In Situ Cell Proliferation Kit, Fluos (Roche). $1.5 \times 10^{4}$ and $1.5 \times 10^{5}$ ENCCs were seeded per slide chamber and $35-\mathrm{mm}$ culture plate, respectively. The cells were treated with either human recombinant Shh at $2 \mu \mathrm{g} / \mathrm{ml}$ or human recombinant GDNF at $100 \mathrm{ng} / \mathrm{ml}$ (PeproTech) or in combination for 72 hours. For the signaling studies, the cells were also treated with Shh inhibitor, cyclopamine $(10 \mu \mathrm{g} / \mathrm{ml}$, Calbiochem); Shh agonist, purmorphamine (1 $\mu \mathrm{M}$, Calbiochem); or Notch inhibitor, DAPT (10 mM, Calbiochem) alone or in combination with Shh or GDNF. BrdU was added 16 hours before the termination of culture. Cells in $35-\mathrm{mm}$ culture plates were then harvested, cytospun onto glass slides, and subjected to immunocytochemistry. Cells were fixed, blocked, and incubated with anti-Ret antibody (1:200, Neuromics). The immunosignal was detected using anti-goat IgG conjugated with Texas Red or Alexa Fluor 680 (Invitrogen). Genomic DNA was denatured in $4 \mathrm{~N} \mathrm{HCl}$ ( 5 minutes at room temperature). After PBS washing, the cultures were incubated with fluorescein-conjugated (1:50, Roche) or monoclonal anti-BrdU (1:50, DAKO) antibodies. The latter required the use of the secondary antibody conjugated with Texas Red for detection. Slides were washed in PBS and mounted with aqueous mounting media with DAPI. The cell proliferation rate was measured by counting the Ret/BrdU double-positive cells against the total number of Ret-positive cells. For each treatment group, a minimum of 9 random fields under $\times 200$ magnification with at least 250 cells in total per $35-\mathrm{mm}$ well was photographed for cell counting. The values reported in bar charts represent the mean \pm SEM of 2 wells (i.e., 18 random fields) and 3 independent experiments were performed.

\section{TUNEL}

Apoptotic cells in ENCC culture and embryos were analyzed on a cytospin preparation of ENCC and paraffin sections, respectively, using the In Situ Cell Death Detection Kit, Fluorescein (Roche), following the manufacturer's protocol.

\section{Immunofluorescence studies}

Immunocytochemistry. To determine the lineage potential of ENCCs after deletion of Ptch1, we analyzed differentiation of adenovirus-infected Ptch1f/f ENCCs. Cells were plated at clonal density, subjected to virus infection for 72 hours, and then treated with GDNF, Dll1 (R\&D Systems), or Notch inhibitor and cultured for an additional 3-10 days. Neuronal lineage commitment was monitored based on expression of TH using rabbit anti-TH antibody (1:1,000, Imgenex) or Tuj1 with mouse anti-Tuj1 antibody (1:200, Chemicon), while the glial differentiation was detected by staining with antibodies specific for $S 100 \beta$ (rabbit 1:200, Sigma-Aldrich) or GFAP (1:200, DAKO); the immunosignal was then detected using the secondary antibody conjugated with Texas Red. All cultures were counterstained with DAPI (blue) to detect nuclei. Numbers of $\mathrm{TH}^{+}, \mathrm{Tuj}^{+}$, and $\mathrm{S} 100 \beta^{+}$cells were counted over the total number of cells (DAPI) in both control (Ad-GFP) and mutant (Ad-GFP-Cre) groups, with or without treatment. For each treatment group, a minimum of 9 random fields under $\times 200$ magnification with at least 250 cells in total per $35-\mathrm{mm}$ well was photographed for cell counting. The values reported in bar charts represent the mean \pm SEM of 2 wells (i.e., 18 random fields), and the experiments were repeated 3 times.

Immunohistochemistry. For section immunohistochemistry, embryos were fixed in $4 \%$ PFA in PBS at $4^{\circ} \mathrm{C}$, dehydrated, and embedded in paraffin or cryoprotected in $30 \%$ sucrose in PBS at $4{ }^{\circ} \mathrm{C}$ and embedded in OCT compound (Tissue-Tek). The sections were blocked in PBS containing 10\% normal goat serum (DAKO) for 1 hour at room temperature and then incubated overnight at $4^{\circ} \mathrm{C}$ in a mixture of the anti-p $75^{\mathrm{NTR}}(1: 2,000$, Chemicon) and anti-Ki67 (1:100, BD Pharmingen). Cryosections were used for the detection of Hes-1 expression with rabbit anti-Hes-1 antibody (1:100, a gift from Tetsuo Sudo, Toray Industries Inc., Kanagawa, Japan). After washing, the immunosignals were then detected using the secondary antibody conjugated with Texas Red or FITC.

For double staining using immunohistochemistry and in situ hybridization, paraffin sections were hybridizated with digoxigenin-labeled (DIG-labeled) antisense cRNA probes (Roche). The hybridized probes were detected using anti-DIG antibody and amplified by the Renaissance TSA Biotin System (PerkinElmer) according to manufacturer's protocol. These sections were then incubated with anti-p $75^{\text {NTR }}$ (Chemicon) for the detection of ENCCs, followed by a goat anti-rabbit IgG secondary antibody conjugated with Texas Red (1:200, Molecular Probes). The signals were then detected by streptavidin-fluorescein conjugate. Sections were counterstained with DAPI and mounted with aqueous mounting media. ENCCs and tissue sections were photographed using a Nikon Eclipse E600 microscope, with Sony digital camera DSM1200F, under fluorescence illumination.

\section{Whole-mount in situ hybridization}

In situ hybridization was used to examine the expression of Fabp7 (NM 021272, GenScript) in embryonic guts. E12.5 embryonic guts were dissected out of control (Ptch1f/f) and mutant (Ptch1fff, bIII-3a-Cre) embryos, fixed with $4 \%$ PFA, and cryoprotected with $30 \%$ sucrose. The whole embryonic guts were hybridized with DIG-labeled RNA probe encoding the coding region of Fabp7, following the standard protocol (34). The signals were detected with BM Purple AP Substrate (Roche). The embryonic guts were then sectioned and photographed. 


\section{Immunoblots}

To examine the activation of cellular signaling, cells were treated with drugs. Cells from three $35-\mathrm{mm}$ wells were collected and then lysed with cell lysis buffer containing $20 \mathrm{mM}$ Tris ( $\mathrm{pH} 7.5$ ), $150 \mathrm{mM} \mathrm{NaCl}, 1 \mathrm{mM}$ EDTA, $1 \mathrm{mM}$ EGTA, 1\% Triton X-100, $2.5 \mathrm{mM}$ sodium pyrophosphate, $1 \mathrm{mM} \beta$ glycerophosphate, $1 \mathrm{mM} \mathrm{Na}_{3} \mathrm{VO}_{4}, 1 \mu \mathrm{g} / \mathrm{ml}$ leupeptin, and $1 \mathrm{mM}$ phenylmethanesulfonyl fluoride. Cell lysates containing $20 \mu \mathrm{g}$ total protein were separated on $8 \%-10 \%$ SDS-polyacrylamide gels and blotted onto nitrocellulose membranes. The membranes were then incubated with polyclonal antibodies against Gli1 (1:3,000, Abcam), Gli3 (1:1,000, Santa Cruz Biotechnology Inc.), Patched-1 (1:500, R\&D Systems), and NICD (1:1,000, Millipore). The same membranes were stripped with stripping buffer $(100 \mathrm{mM}$ 2-mercaptoethanol, $2 \%$ sodium dodecyl sulfate, and $62.5 \mathrm{mM}$ Tris- $\mathrm{HCl}, \mathrm{pH}$ 6.7), blocked, and reprobed with a 1:1,000 dilution of anti- $\beta$-actin monoclonal antibody (Millipore) to ensure equal loading of cell protein per lane. All blots were incubated with 1:5,000 dilutions of secondary horseradish peroxidase-conjugated anti-mouse or anti-rabbit antibody (Amersham Pharmacia Biotech.). Antibody-bound proteins were visualized using a chemiluminescence system (Amersham Pharmacia Biotech). The representative pictures of at least 3 independent assays are shown.

\section{RT-PCR}

Total RNA was isolated from ENCCs by the RNeasy Mini Kit (Qiagen) and reverse transcribed in $20 \mu \mathrm{l}$ reaction system using the SuperScript RNA Amplification System (Invitrogen), in accordance with the manufacturer's instructions. PCR reactions were performed using specific primers (listed in Supplemental Table 3). Results were normalized and expressed relative to the internal control, actin.

\section{Quantitative RT-PCR}

RNA for RT-PCR was extracted from mouse embryonic guts or ENCCs using the RNeasy Mini Kit (Qiagen) and reverse transcribed in $20 \mu \mathrm{l}$ reaction mix as above. Quantitative PCR was performed in the Cybergreen reaction mix (Applied Biosystems), which consisted of $1 \times$ mastermix and forward and reverse primers. The reaction mix $(18 \mu \mathrm{l})$ was aliquoted into tubes, and $2 \mu \mathrm{l}$ cDNA was added. Triplicated $20 \mu \mathrm{l}$ samples plus positive (HTB11 cDNA, a human neuroblastoma cell line, or ENCC cDNA) and negative (water) controls were placed in a PCR plate, and wells were sealed

1. Heanue TA, Pachnis V. Enteric nervous system development and Hirschsprung's disease: advances in genetic and stem cell studies. Nat Rev Neurosci. 2007;8(6):466-479

2. Ingham PW, McMahon AP. Hedgehog signaling in animal development: paradigms and principles. Genes Dev. 2001;15(23):3059-3087.

3. Jiang J, Hui CC. Hedgehog signaling in development and cancer. Dev Cell. 2008;15(6):801-812.

4. Lu QR, et al. Sonic hedgehog--regulated oligodendrocyte lineage genes encoding bHLH proteins in the mammalian central nervous system. Neuron. 2000;25(2):317-329.

5. Wall DS, et al. Progenitor cell proliferation in the retina is dependent on Notch-independent Sonic hedgehog/Hes1 activity. J Cell Biol. 2009; 184(1):101-112.

6. Yang ZJ, et al. Medulloblastoma can be initiated by deletion of Patched in lineage-restricted progenitors or stem cells. Cancer Cell. 2008;14(2):135-145.

7. McMahon AP, Ingham PW, Tabin CJ. Developmental roles and clinical significance of hedgehog signaling. Curr Top Dev Biol. 2003;53:1-114.

8. Nieuwenhuis E, Hui CC. Hedgehog signaling and congenital malformations. Clin Genet. 2005;67(3):193-208.

9. Ramalho-Santos M, Melton DA, McMahon AP. Hedgehog signals regulate multiple aspects with optical caps. The PCRs were carried out using an ABI Prism 7900 (Applied Biosystems). All primers sequences are listed in Supplemental Table 3. Data were analyzed and processed using Sequence Detector version 1.6.3 (Applied Biosystems) in accordance with the manufacturer's instructions. Primers (concentration and annealing temperature) were optimized, and the linearity of the results was validated in serial dilution of a cDNA pool. Results were expressed relative to a positive standard (cDNA obtained from HTB11 or a single sample of ENCCs). 18S was used as the internal control. The values reported in bar charts represent the mean \pm SEM, and the experiments were repeated in independent assays.

\section{Statistics}

The differences among multiple treatment groups were analyzed with a 2-sided unpaired Student $t$ test or 1-way ANOVA, followed by Tukey post-hoc test using GraphPad Prism 5 (GraphPad Software). A $P$ value of less than 0.05 was interpreted to represent a statistically significant difference. All experiments were replicated at least 3 times, and data are shown as mean \pm SEM.

\section{Acknowledgments}

This work was supported by a seed funding grant for basic research from the University of Hong Kong to E.S.W. Ngan and by research grants HKU775710 and HKU773909 to E.S.W. Ngan and HKU765407, HKU775907, and HKU752806 to M.M. GarciaBarceló, P.K.H. Tam, and V.C.H. Lui, respectively, from the Hong Kong Research Grants Council. The authors thank Robin LovellBadge (MRC National Institute for Medical Research, London, United Kingdom), S.Y. Tsai (Baylor College of Medicine, Houston, Texas, USA), and Kathryn Cheah (University of Hong Kong, Hong Kong, China) for critical reading of the manuscript.

Received for publication May 18, 2010, and accepted in revised form June 22, 2011.

Address correspondence to: Elly S.W. Ngan, Department of Surgery, University of Hong Kong, Pokfulam, Faculty of Medicine Building, 21 Sassoon Road, Hong Kong, SAR, China. Phone: 852.2819.9631; Fax: 852.2816.9621; E-mail: engan@hku.hk. of gastrointestinal development. Development. 2000;127(12):2763-2772.

10. Okamura Y, Saga Y. Notch signaling is required for the maintenance of enteric neural crest progenitors. Development. 2008;135(21):3555-3565.

11. Morrison SJ, et al. Transient Notch activation initiates an irreversible switch from neurogenesis to gliogenesis by neural crest stem cells. Cell. 2000;101(5):499-510.

12. Wakamatsu Y, Maynard TM, Weston JA. Fate determination of neural crest cells by NOTCHmediated lateral inhibition and asymmetrical cell division during gangliogenesis. Development. 2000;127(13):2811-2821.

13. Bray SJ. Notch signalling: a simple pathway becomes complex. Nat Rev Mol Cell Biol. 2006;7(9):678-689.

14. Garcia-Barcelo MM, et al. Genome-wide association study identifies NRG1 as a susceptibility locus for Hirschsprung's disease. Proc Natl Acad Sci U S A. 2009;106(8):2694-2699.

15. Fu M, Lui VC, Sham MH, Pachnis V, Tam PK. Sonic hedgehog regulates the proliferation, differentiation, and migration of enteric neural crest cells in gut. J Cell Biol. 2004;166(5):673-684.

16. Miele L, Miao H, Nickoloff BJ. NOTCH signaling as a novel cancer therapeutic target. Curr Cancer Drug Targets. 2006;6(4):313-323.

17. Ellis T, et al. Patched 1 conditional null allele in mice. Genesis. 2003;36(3):158-161.

18. Lui VC, et al. Perturbation of hoxb5 signaling in vagal neural crests down-regulates ret leading to intestinal hypoganglionosis in mice. Gastroenterology. 2008;134(4):1104-1115

19. Fernandes KJ, et al. A dermal niche for multipotent adult skin-derived precursor cells. Nat Cell Biol. 2004;6(11):1082-1093.

20. Toma JG, McKenzie IA, Bagli D, Miller FD. Isolation and characterization of multipotent skinderived precursors from human skin. Stem Cells. 2005;23(6):727-737.

21. Artavanis-Tsakonas S, Rand MD, Lake RJ. Notch signaling: cell fate control and signal integration in development. Science. 1999;284(5415):770-776.

22. Baetge G, Gershon MD. Transient catecholaminergic (TC) cells in the vagus nerves and bowel of fetal mice: relationship to the development of enteric neurons. Dev Biol. 1989;132(1):189-211.

23. Young HM, Turner KN, Bergner AJ. The location and phenotype of proliferating neural-crest-derived cells in the developing mouse gut. Cell Tissue Res. 2005;320(1):1-9.

24. Furukawa T, Mukherjee S, Bao ZZ, Morrow EM, Cepko CL. rax, Hes1, and notch1 promote the formation of Muller glia by postnatal retinal progenitor cells. Neuron. 2000;26(2):383-394.

25. Gaiano N, Nye JS, Fishell G. Radial glial identity is 
promoted by Notch 1 signaling in the murine forebrain. Neuron. 2000;26(2):395-404.

26. Jadhav AP, Cho SH, Cepko CL. Notch activity permits retinal cells to progress through multiple progenitor states and acquire a stem cell property. Proc Natl Acad Sci U S A. 2006;103(50):18998-19003.

27. Mizutani K, Saito T. Progenitors resume generating neurons after temporary inhibition of neurogenesis by Notch activation in the mammalian cerebral cortex. Development. 2005;132(6):1295-1304.

28. Thompson B, ed. Canonical Correlation Analysis: Uses And Interpretation. Beverly Hills, California, USA
Sage Publications; 1984.

29. Hahn LW, Ritchie MD, Moore JH. Multifactor dimensionality reduction software for detecting gene-gene and gene-environment interactions. Bioinformatics. 2003;19(3):376-382.

30. Price AL, Patterson NJ, Plenge RM, Weinblatt ME, Shadick NA, Reich D. Principal components analysis corrects for stratification in genome-wide association studies. Nat Genet. 2006;38(8):904-909.

31. Jiang X, Rowitch DH, Soriano P, McMahon AP, Sucov HM. Fate of the mammalian cardiac neural crest. Development. 2000;127(8):1607-1616.
32. Ngan ES, et al. Prokineticin-1 modulates proliferation and differentiation of enteric neural crest cells. Biochim Biophys Acta. 2007;1773(4):536-545.

33. Ngan ES, et al. Prokineticin-1 (Prok-1) works coordinately with glial cell line-derived neurotrophic factor (GDNF) to mediate proliferation and differentiation of enteric neural crest cells. Biochim Biophys Acta. 2008;1783(3):467-478.

34. Rosen B, Beddington RS. Whole-mount in situ hybridization in the mouse embryo: gene expression in three dimensions. Trends Genet. 1993;9(5):162-167. 